\title{
Expression of Concern: Synchronization in asymmetrically coupled networks with homogeneous oscillators [Phys. Rev. E 103, 022206 (2021)]
}

(Received 29 July 2021; published 17 September 2021)

DOI: 10.1103/PhysRevE.104.039902

This paper by Antonio Palacios includes several inaccurate statements with respect to two previous papers by Takashi Nishikawa and Adilson E. Motter [1] and Yuanzhao Zhang, Takashi Nishikawa, and Adilson E. Motter [2].

In the abstract of the paper it is stated that Refs. [1] and [2] had reported "that in networks with asymmetrically coupled oscillators, synchronization can only be found to be stable when the oscillators are heterogenous or nonidentical." This claim is then apparently supported by several quotes from Ref. [1] which are misleading or incomplete. No such general statement is made in Ref. [1], which in contrast states: "As in other systems subject to symmetry breaking, such symmetric states are not always stable."

Thus, also the statement in this paper, "In this manuscript, it is proven, mathematically, that the conclusions in those works are incorrect," is inaccurate since these conclusions were quoted and interpreted incorrectly.

Michael Thoennessen Editor in Chief

[1] Takashi Nishikawa and Adilson E. Motter, Phys. Rev. Lett. 117, 114101 (2016).

[2] Yuanzhao Zhang, Takashi Nishikawa, and Adilson E. Motter, Phys. Rev. E 95, 062215 (2017). 